Reviews

\title{
Needle-type Extraction Device Designed for Rapid and Sensitive Analysis in Gas Chromatography
}

\author{
Ikuo UETA* and Yoshihiro SAITO**† \\ *Department of Applied Chemistry, University of Yamanashi, Kofu 400-8511, Japan \\ **Department of Environmental and Life Sciences, Toyohashi University of Technology, Toyohashi 441-8580, \\ Japan
}

\begin{abstract}
A needle-type extraction device is one of the promising sample preparation devices for the gas chromatographic (GC) analysis of various organic compounds. The most remarkable advantage of this device is a simple and rapid desorption of the analytes by direct insertion of an extraction needle into a conventional GC injection port. In this review, fundamental aspects of the needle-type extraction devices and its applications for the determination of trace organic compounds, especially volatile organic compounds (VOCs), in several sample matrices are described.
\end{abstract}

Keywords Sample preparation, extraction needle, volatile organic compounds, gas chromatography

(Received September 3, 2013; Accepted September 6, 2013; Published January 10, 2014)

\begin{tabular}{lcll}
1 Introduction & \multirow{2}{*}{105} & Aqueous Samples & 108 \\
2 Fundamentals of the Needle-type Extraction & & 5 Conclusion and Future Prospects & 109 \\
Device & 106 & 6 Acknowledgements & 109 \\
3 Determination of VOCs in Gaseous Samples & 106 & 7 References & 109 \\
4 Extraction of Organic Compounds from & & &
\end{tabular}

\section{Introduction}

Sample preparation is an important step in modern chemical analysis, and it directly affects the accuracy, precision and sensitivity of the entire analytical method. However, this step is normally a time-consuming process, and therefore, development of a rapid and accurate sample preparation technique has been regarded as one of the most important objectives in the field of analytical science and the related research area., ${ }^{1,2}$
Miniaturization of the sample preparation has also been widely studied in liquid-phase separations ${ }^{3-10}$ using precisely controlled extraction media such as prepared by molecular shape recognition concept ${ }^{11-16}$ or computer simulation of interaction. ${ }^{17-20}$

A large number of sample preparation methods have been developed for the extraction of volatile organic compounds (VOCs) in gas chromatographic (GC) analysis. For example, the extraction of VOCs using charcoal tubes is a traditional sample preparation technique, where the extracted VOCs were desorbed by an organic solvent, such as carbon disulfide, and

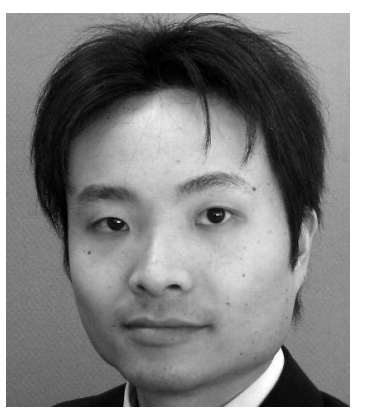

Ikuo UETA received his Ph.D. degree in March 2010 from Toyohashi University of Technology, and then worked as a JSPS postdoctoral researcher at the university until being appointed as an Assistant Professor in the Department of Applied Chemistry, University of Yamanashi, in October 2010. He received the Young Scientist Award from the Society for Chromatographic Sciences in 2012. His research interests include the development and application of novel miniaturized extraction devices for sensitive analysis in gas chromatography and liquid chromatography, including sensitive determination of volatile organic compounds in environmental air/water samples and also human breath samples.

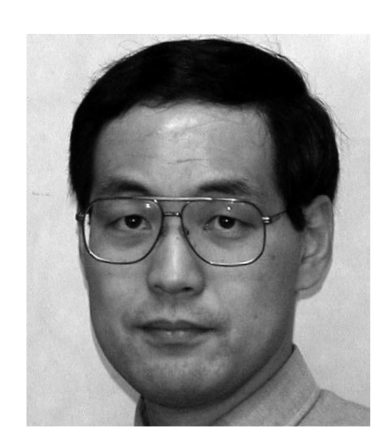

Yoshihiro SaITo received his Ph.D degree in 1996 from Toyohashi University of Technology. After spending a period of two years as a postdoctoral researcher in the US, he has returned to Toyohashi as a Research Associate, and was then promoted to Assistant Professor in 2000 and Associate Professor in 2008. $\mathrm{He}$ received the Young Scientist Award from The Society for Chromatographic Sciences and the Tokai Chemical Industry Award. He has been an Associate Editor of Analytical Sciences and has also served on the Editorial Advisory Board of Chromatographia. He is the author or co-author of over 100 research papers, especially related to the development of microscale analytical systems and their applications.

$\dagger$ To whom correspondence should be addressed.

E-mail: saito@ens.tut.ac.jp 


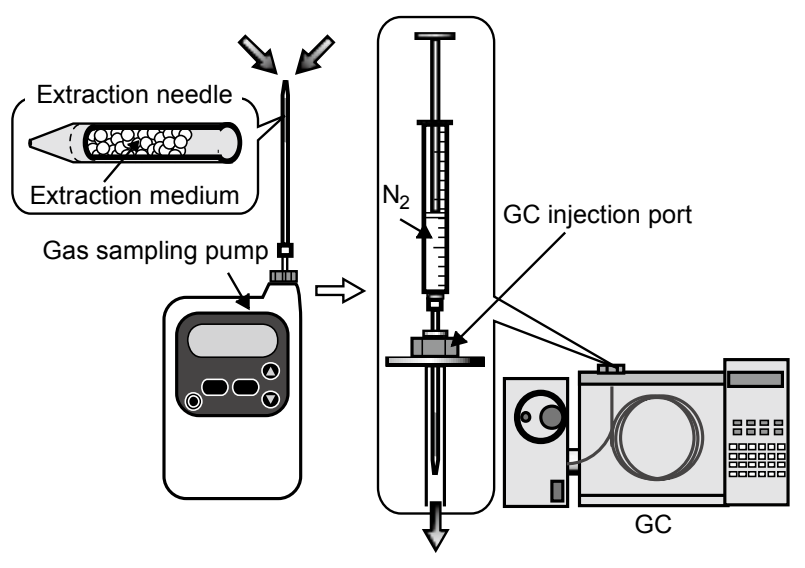

Fig. 1 Typical extraction and desorption procedures of gaseous VOCs with the needle-type extraction device.

the resulting solution was analyzed by GC. The technique has been widely employed; however, there are some disadvantages, such as multi-step and complicated procedures along with a requirement for a long analysis time. The thermal desorption (TD) method is an alternative sample preparation technique of the above solvent desorption method. The conventional TD process enables a sensitive and fully-automated analysis, although relatively expensive specially-designed instruments are necessary. ${ }^{21-23}$ Solid-phase microextraction (SPME) is a simple and solvent-free sample preparation technique, ${ }^{24,25}$ which is based on the equilibrium between the sample matrix and the extraction phase that is immobilized on a solid support. This method has been widely applied to the analysis of VOCs; however, the extraction medium consisted of a fused-silica rod, which is quite fragile. The possibility of a problematic carryover must also be considered in the repeatable use.

The needle-type extraction device is one of the effective sample preparation methods for the analysis of VOCs..$^{26-28}$ The fundamental concept of the needle-based extraction method was mainly developed in the early 2000s by several research groups, and many publications have appeared. ${ }^{29}$ The device typically contains particulate extraction material(s) in a thin-wall stainless-steel needle. For specific target analyte(s), the type of sorbent packed in the needle can be easily optimized on the basis of relatively quick preliminary experiments, allowing a wide range of applications to the GC analysis of VOCs.

This review mainly focuses on the determination of VOCs by needle-type extraction devices in GC. Fundamental aspects of the needle-type extraction devices is reviewed along with the applications of several types of extraction needles that contain different types of sorbents for an effective extraction of respective target analyte(s) from both gaseous and aqueous samples.

\section{Fundamentals of the Needle-type Extraction Device}

In the needle-type extraction device, an extraction sorbent is packed inside of a stainless-steel needle. As extraction sorbents, porous polymer beads or activated carbon (AC) based particles have typically been employed for effective extraction/desorption of VOCs. ${ }^{30}$ The diameter of the particulate medium is normally in the range from 150 to $250 \mu \mathrm{m}$. In order to effectively extract a wide range of VOCs, a multi-bed-type extraction needle, containing more than one sorbents has also been introduced..$^{30}$ Most of the needle-type extraction devices have typically been designed for the active sampling of gaseous samples. The analytes are extracted on the packed sorbent when a gaseous sample is passed through the sorbent-packed section of the extraction needle. For the extraction of gaseous samples, the sample was introduced from the tip side of the needle typically using a syringe or pump. The limit of detection (LOD) of the analytes depends on the sampling volume, having a typical value of from $10 \mathrm{~mL}$ to $1 \mathrm{~L}$. A sampling flow rate of lower than $20 \mathrm{~mL} / \mathrm{min}$ is often preferable for the effective extraction of most air samples. ${ }^{31,32}$

After the extraction of analytes, the needle is directly inserted into a GC injection port. The extracted analytes can then be thermally desorbed in the heated GC injection port, typically without a desorption solvent. . $^{31,33,34}$ For desorption of the extracted analytes from the sorbent, there are two types of desorption methods: one uses carrier gas flow as the desorption gas, ${ }^{32-37}$ while another way is not using a carrier gas. When not using a carrier gas, the flow of an inert gas, supplied from a gas tight syringe, was used as the desorption gas. ${ }^{31,38-44}$ More recently, a new desorption method using a water vapor stream, generated from packed alumina, was also reported. ${ }^{45}$ Among these desorption techniques, the first method is suitable for automation; however, it often requires a special instrument or liner. The second method is a simple process, and it does not require any specific instrument or modification of GC, as typically shown in Fig. 1.

Because of the above simple extraction/desorption processes using a needle-type extraction device, the method has been regarded as being a fast, sensitive, robust, and repeatable extraction method. The needle-type extraction devices have been employed, especially for the analysis of VOCs in gaseous samples, ${ }^{39-43}$ and the devices have been applied to biomedical analysis, such as the determination of VOCs in human breath samples. ${ }^{44,45}$ The determination of VOCs in aqueous samples by needle-type extraction device was also reported. ${ }^{36-38,45-47}$

\section{Determination of VOCs in Gaseous Samples}

Berezkin et al. reported on needle-type extraction devices packed with Tenax as the extraction sorbent. ${ }^{48}$ The extraction needle was employed for the determination of benzene and toluene in tobacco smoke, and it was further applied to the determination of benzene, toluene, ethylbenzene and xylenes (BTEX) in aqueous samples. The group of Pawliszyn developed two types of needle extraction devices. ${ }^{33}$ One is a multi-bedtype extraction needle containing polydimethylsiloxane (PDMS), divinylbenzene (DVB) and Carboxen, and another is packed with only Carboxen 1000. The LODs for benzene, ethylbenzene, and xylene using the latter extraction needle were $0.23,2.10$ and $1.12 \mathrm{ng} / \mathrm{L}$, respectively, at a sampling volume of $25 \mathrm{~mL}$. Alonso et al. compared two types of extraction needles packed with Carbopack X and Tenax TA. ${ }^{32}$ They applied the method to the analysis of VOCs in breath, indoor air and outdoor air samples, confirming several advantages of the needle-type extraction device.

To effectively extract several VOCs, Saito et al. developed co-polymer beads of methacrylic acid (MA) and ethylene glycol dimethacrylate (EGDMA) as the extraction medium. ${ }^{31} \mathrm{~A}$ gaseous sample was collected by a vacuum gas sampling device, and the extracted VOCs were determined by a flame ionization detector (FID) in GC. The results clearly indicated satisfactory extraction and desorption performances along with an excellent 
storage performance of the extracted analytes in the extraction needle, typically up to 7 days, at room temperature. The extraction needle was then adapted for the evaluation of third-hand smoking, ${ }^{40}$ where smoking-related VOCs evaporated from several types of fabrics to the environmental air were extracted and determined by mass spectrometry (MS). Third-hand smoking pollution in an automobile was also confirmed. In addition, a novel fire investigation technique was developed with a needle-type extraction device. For the determination of VOCs directly related to fire accelerants, such as gasoline or kerosene, a polymer particle of DVB was introduced as the extraction medium. The needle extraction was successfully applied to the determination of VOCs in simulated fires, as shown in Fig. $2 .^{41}$

A novel double-bed-type extraction needle was developed for the determination of VOCs in indoor air environments. ${ }^{42}$ In this study, indoor air samples including a wide range of VOCs were collected for $30 \mathrm{~min}$ using a sampling pump. To determine an optimum extraction medium, several types of particulate sorbents were tested, and a double-bed-type extraction needle
(A)

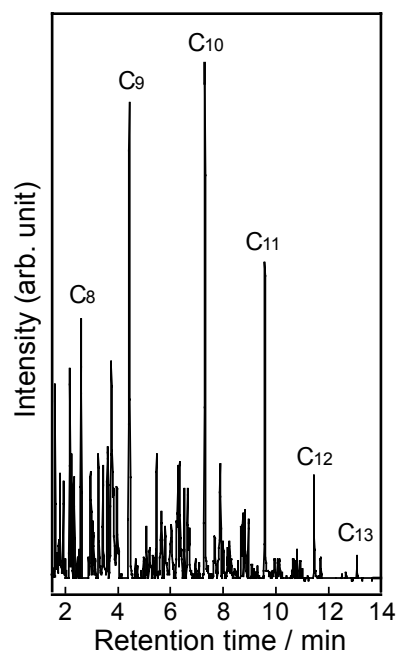

(B)

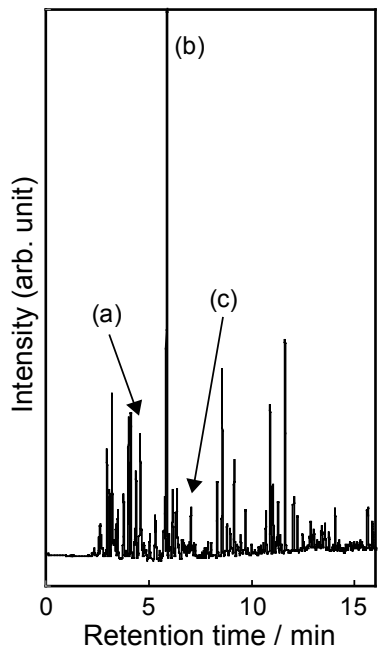

Fig. 2 Typical chromatograms of extracted VOCs in two simulated fire scenes. (A) Simulation of an arson fire and (B) simulation of misfueling of gasoline into kerosene heater. Peaks: (a) 1-ethyl-2methylbenzene, (b) 1,2,4-trimethylbenzene, (c) 1,2,3-trimethylbenzene. containing DVB and AC particles showed excellent extraction and desorption performances for all of the investigated VOCs. The proposed extraction method was compared to the corresponding conventional solvent extraction method in the analysis of real indoor air samples. This double-bed-type extraction needle was then applied to an evaluation of the indoor air environment in school facilities, ${ }^{43}$ suggesting the advantages of the needle-type extraction device for assessing indoor air environments.

On the basis of the above successful extraction performances of the needle-type extraction device for a wide range of VOCs having a different volatility and/or polarity, the extraction needle has been successfully applied to the determination of VOCs in human breath. Ueta et al. determined the breath acetone concentration for healthy subjects and type- 2 diabetic patients by using a MA/EGDMA-packed extraction needle in the sample preparation step prior to the analysis in GC-MS. ${ }^{44}$ For the diagnosis of diabetes, the glucose concentration and glycosylated hemoglobin level in blood, $\mathrm{HbA} 1 \mathrm{c} \%$, have been used along with the concentration of the ketone bodies in urine. $\mathrm{HbA} 1 \mathrm{c} \%$ is the percentage of hemoglobin-sugar complex relative to total hemoglobin in blood, and that well represents an average amount of glucose level in blood over about the last two to three months. A higher $\mathrm{HbA} 1 \mathrm{c} \%$ value means a higher glucose concentration in blood, although the value is not affected by a short-term variation that might be induced by a meal, or other miscellaneous factors. The results well demonstrated that there is a clear correlation between the concentration of breath acetone and $\mathrm{HbA} 1 \mathrm{c} \%$ for type- 2 diabetic patients. In addition, a high correlation between the breath and urine acetone concentrations during a fasting test was also confirmed for healthy subjects.

Mieth et al. introduced a multibed-type extraction needle for the on-site sampling of breath samples, ${ }^{34}$ and applied the needle to the sample preparation of breath VOCs for subsequent analysis in a heart-cut GC/MS, and also comprehensive two dimensional GC-time-of-flight mass spectrometry (GCxGC/ TOF-MS). ${ }^{35}$ Trefz et al. investigated the sample flow rate and sample volume, along with the effect of humidity for breath analysis using a MA/EGDMA-packed needle and a triple-bed extraction needle. ${ }^{49}$ They reported that the triple-bed extraction needle was not affected by the humidity. Determinations of VOCs in gaseous or breath samples by needle-type extraction devices are summarized in Table 1.

In order to extract high-volatile VOCs, several VOCs should be derivatized before chromatographic analysis. With a fiberpacked extraction needle, derivatization reactions were introduced. The extraction needle was packed longitudinally

Table 1 Needle-type extraction device for the determination of VOCs in gaseous samples

\begin{tabular}{|c|c|c|c|c|c|}
\hline Packed sorbent & Analyte & Sampling volume/mL & Detection & $\mathrm{LOD} / \mathrm{ng} \mathrm{L}^{-1}$ & Ref. \\
\hline Tenax & Benzene, toluene & & & & 48 \\
\hline Tenax TA & $10 \mathrm{VOCs}$ & 1000 & MS & $0.002-0.01$ & 32 \\
\hline Carboxen 1000 & Benzene, ethylbenzene, xylene & 25 & FID & $0.23-2.10$ & 33 \\
\hline MA/EGDMA & Hexane, acetone, ethyl acetate, toluene & 50 & FID & 1000 & 31 \\
\hline MA/EGDMA & Tobacco related VOCs & 50 & MS & $0.5-5.0$ & 40 \\
\hline DVB & Fire accelerants related VOCs & 50 & MS & $0.03-0.1$ & 41 \\
\hline DVB/AC & Indoor air VOCs & 600 & MS & $0.004-0.02$ & 42 \\
\hline DVB/AC & VOCs in school facilities & $50 / 600$ & MS & & 43 \\
\hline MA/EGDMA & Breath acetone & 50 & MS & 1.2 & 44 \\
\hline Tenax/Carbopack X/Carboxen 1000 & Breath VOCs & 25 & MS & $0.4-8.3$ & 34 \\
\hline Tenax/Carbopack X/Carboxen 1000 & Breath VOCs & 20 & $\begin{array}{c}\text { MS } \\
\text { TOF-MS }\end{array}$ & & 35 \\
\hline DVB/Carbopack X/Carboxen 1000 & Breath VOCs & $10-100$ & MS & & 49 \\
\hline
\end{tabular}


with a bundle of Zylon filaments $(12 \mu \mathrm{m}$ o.d. $),{ }^{50,51}$ which were also surface-coated with polymeric materials, as illustrated in Fig. 3. Taking advantage of a good heat-resistant property along with the stability to organic solvents and reagents, ${ }^{50-56} \mathrm{a}$ Zylon-packed needle could be employed in the sample preparation process using a derivatization reaction of target compounds. For the extraction of volatile aldehydes and ketones, a derivatization reaction with 2,4-dinitrophyenylhydrazine (DNPH) was introduced ${ }^{57}$ After loading of the DNPH solution into the needle extraction device, a gaseous sample was collected through the fiber-packed needle. During the sampling, analytes were reacted with the derivatization reagent on the packed filaments at room temperature, and the derivatives were simultaneously extracted on the polymer coating of the fiber. The extracted derivatives were then desorbed by injecting acetonitrile and $\mathrm{N}_{2}$ gas through the needle in the $\mathrm{GC}$ injector. Volatile aldehydes were also derivatized by $O$ - $(2,3,4,5,6-$ pentafluorobenzyl)hydroxylamine (PFBHA) using the fiberpacked needle. ${ }^{39}$ This method was further applied to the determination of gaseous ethylene oxide (EO), ${ }^{58}$ where an aqueous solution of $\mathrm{HBr}$ was used for the derivatization of $\mathrm{EO}$

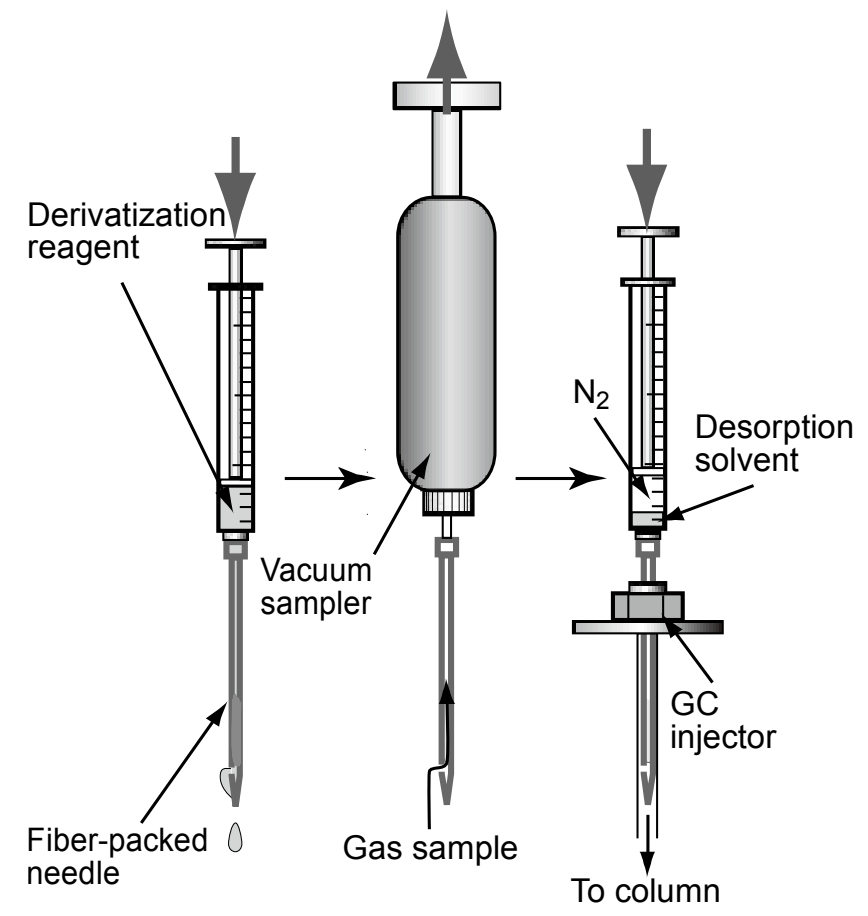

Fig. 3 Determination of VOCs with derivatization reaction using a fiber-packed needle. in the needle. As the desorption solvent, methanol was used for an effective desorption of the extracted derivative, 2-bromoethanol, from the needle. Successful applications to the analysis of EO in tobacco smoke and automobile exhaust were demonstrated in the report.

\section{Extraction of Organic Compounds from Aqueous Samples}

The amounts of organic compounds, including VOCs, in tap water or drinking water, are typically on the order of $\mu \mathrm{g} / \mathrm{L}$ to ng/L. To determine trace levels of organic compounds in these water samples, an effective sample preparation is needed. The extraction of the VOCs in aqueous samples by a needle-type extraction device showed significantly higher extraction amounts than SPME. ${ }^{59}$ The representative publications for the determination of organic compounds in aqueous samples by needle-type extraction device are tabulated in Table 2. For the extraction of BTEX in aqueous samples, a novel extraction needle was developed. ${ }^{45}$ An extraction needle contained Porapak $\mathrm{Q}$ and wet alumina; they could work as the sorbent and the source of water vapor for the desorption of the extracted target analytes, respectively. In this method, aqueous samples were directly passed through the needle. BTEX in aqueous samples was also analyzed by a purge and trap (PT) technique using a Carbopack X-packed extraction needle. ${ }^{46}$ Crom et al. also reported the determination of BTEX in aqueous samples using a Carbopack X-packed needle, where several experimental parameters were systematically optimized. ${ }^{36}$ Additionally, the same research group compared two techniques, headspace and PT, for the analysis of VOCs in aqueous samples using a needle extraction device. They concluded that PT analysis showed a better sensitivity. ${ }^{37}$ Lou et al. determined formic and acetic acids in aqueous sample using a DVB-packed extraction needle in GC-FID. ${ }^{38}$

Ueta et al. demonstrated an effective analysis of $23 \mathrm{VOCs}$ in aqueous samples by PT with a multibed-type extraction needle, as illustrated in Fig. $4 .{ }^{47}$ As the extraction medium, DVB, AC particles, and carbon molecular sieve (CMS) particles were used. The technique does not require any desorption solvent for the analysis of VOCs in aqueous samples. The limit of quantifications of all the investigated VOCs reached 10-times lower than its reference values. The method was applied to the analysis of real tap-water samples, and some trace VOCs were successfully detected.

A fiber-packed extraction needle was applied to the dynamic extraction of aromatic compounds in aqueous samples. ${ }^{60}$ The extraction was made by pumping the sample solution into the extraction needle, and the subsequent desorption was carried out using a desorption solvent of dichloromethane. The fiber-

Table 2 Needle-type extraction device for the determination of organic compounds in aqueous samples

\begin{tabular}{|c|c|c|c|c|c|c|}
\hline Packed sorbent & Analyte & Sampling method & Sample volume/mL & Detection & $\mathrm{LOD} / \mathrm{ng} \mathrm{L}^{-1}$ & Ref. \\
\hline Porapak Q/alumina & BTEX & Dynamic extraction & 5 & FID & $430-830$ & 45 \\
\hline Carbopack X & BTEX & PT & 50 & FID & $50-70$ & 46 \\
\hline Carbopack X & BTEX & Static HS & & FID & $10000-25000$ & 36 \\
\hline Tenax TA/Carboxen 1000 & $18 \mathrm{VOCs}$ & $\begin{array}{l}\text { Static HS } \\
\text { PT }\end{array}$ & 5 & MS & $10-60$ & 37 \\
\hline DVB & Formic and acetic acids & PT & 20 & FID & $87200-234800$ & 38 \\
\hline DVB/AC/CMS & 23 VOCs & PT & 20 & MS & $10-300$ & 47 \\
\hline Polymer-coated fiber & Aromatic compounds & Dynamic extraction & 0.16 & FID & $200-2000$ & 60 \\
\hline Polymer-coated fiber & Bisphenol A & Dynamic extraction & 0.16 & MS & $<500$ & 61 \\
\hline
\end{tabular}




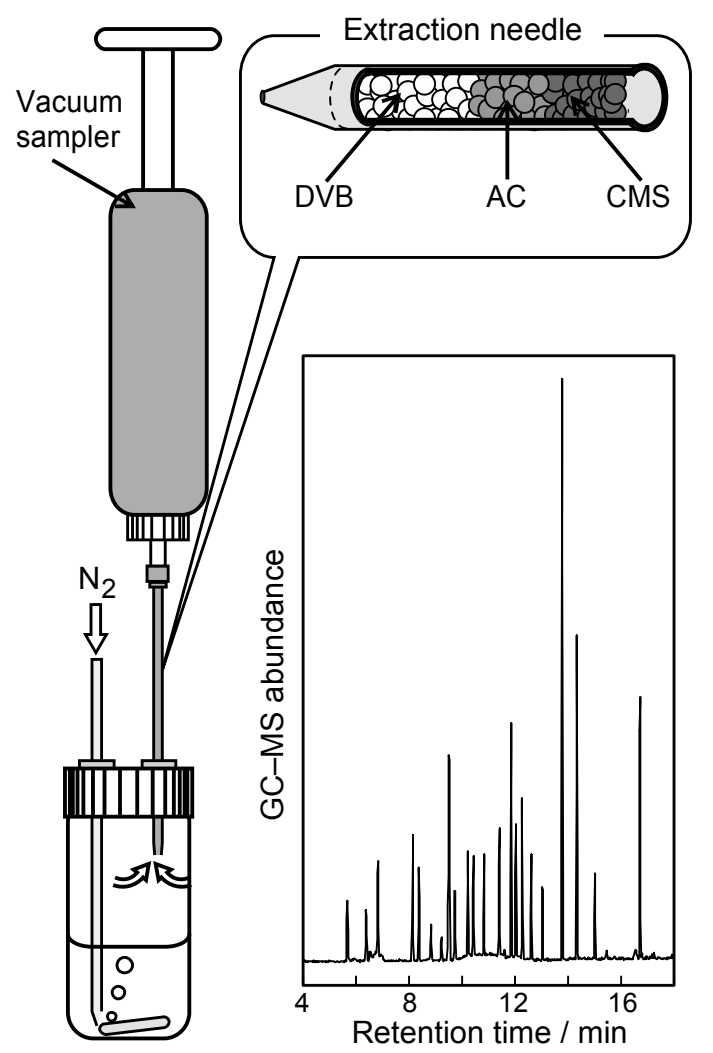

Fig. 4 PT analysis of VOCs in an aqueous sample by a multibedtype needle extraction device.

packed needle was also employed for the determination of bisphenol $\mathrm{A}$ in water samples using the derivatization reaction with $\quad \mathrm{N}, \mathrm{O}$-bis(trimethylsilyl)trifluoroacetamide $\quad$ (BSTFA). ${ }^{61}$ After the extraction of bisphenol A onto the polymer-coated fiber by pumping aqueous solution into the needle, the remaining water in the needle was completely eliminated by $\mathrm{N}_{2}$ gas flow, aspiration by a vacuum pump, and heating. Then, BSTFA was introduced into the needle, and reacted with the analyte by heating. The derivative was successfully desorbed with a desorption solvent of dichloromethane.

\section{Conclusion and Future Prospects}

The development of a miniaturized and effective sample preparation method is one of the important objectives in the field of analytical science; ${ }^{62,63}$ it should be continuously studied to construct a simple and effective analytical method in the next generation. The extraction of organic compounds by a needletype extraction device showed a simple and sensitive extraction method, and actually several applications have been reported, including the sample preparation of gaseous and aqueous samples. Further applications of the extraction needle could be performed, such as the determination of organic compounds in food and drink, soil or biomedical samples. In addition, the needle-type extraction device could be hyphenated with other types of separation techniques, such as liquid chromatography or capillary electrophoresis.

Techniques developed related to the needle-type extraction devices include a precise packing process of the fine particles into the capillary tube, which have been applied to the development of packed-capillary columns. ${ }^{64-66}$ A wide variety of additional applications of the needle-type extraction devices coupled with the packed-capillary column will be expected, ${ }^{67-70}$ along with further developments of miniaturized separation techniques, while offering many advantageous features.

\section{Acknowledgements}

Part of this study was financially supported by Grants-in-Aid for Scientific Research from the Japan Society for the Promotion of Science (JSPS), Kurita Water and Environment Foundation and the Hibi Science Foundation. The authors acknowledge the technical support of Mr. K. Fujimura and Dr. H. Wada of Shinwa Chemical Industries, Ltd., Kyoto, Japan and Prof. K. Jinno, Toyohashi University of Technology. One of the authors (I. U.) express their gratitude for support from Prof. S. Kawakubo, University of Yamanashi.

\section{References}

1. K. Demeestere, J. Dewulf, B. D. Witte, and H. V. Langenhove, J. Chromatogr., A, 2007, 1153, 130.

2. Y. Saito and K. Jinno, J. Chromatogr., A, 2003, 1000, 53.

3. Y. Saito, Y. Nakao, M. Imaizumi, T. Takeichi, Y. Kiso, and K. Jinno, Fresenius' J. Anal. Chem., 2000, 368, 641.

4. Y. Saito, M. Kawazoe, M. Hayashida, and K. Jinno, Analyst, 2000, 125, 807.

5. Y. Saito, M. Imaizumi, T. Takeichi, and K. Jinno, Anal. Bioanal. Chem., 2002, 372, 164.

6. Y. Saito, Y. Nakao, M. Imaizumi, Y. Morishima, Y. Kiso, and K. Jinno, Anal. Bioanal. Chem., 2002, 373, 81.

7. Y. Saito, M. Nojiri, M. Imaizumi, Y. Nakao, Y. Morishima, H. Kanehara, H. Matsuura, K. Kotera, H. Wada, and K. Jinno, J. Chromatogr., A, 2002, 975, 105.

8. M. Imaizumi, Y. Saito, M. Hayashida, T. Takeichi, H. Wada, and K. Jinno, J. Pharm. Biomed. Anal., 2003, 30, 1801.

9. Y. Saito, M. Imaizumi, K. Ban, A. Tahara, H. Wada, and K. Jinno, J. Chromatogr., A, 2004, 1025, 27.

10. M. Imaizumi, Y. Saito, K. Ban, H. Wada, M. Hayashida, and K. Jinno, Chromatographia, 2004, 60, 619.

11. Y. Saito, K. Jinno, J. J. Pesek, Y.-L. Chen, G. Luehr, J. Archer, J. C. Fetzer, and W. R. Biggs, Chromatographia, 1994, 38, 295.

12. Y. Saito, H. Ohta, H. Nagashima, K. Itoh, K. Jinno, and J. J. Pesek, J. Microcolumn Sep., 1995, 7, 41.

13. Y. Saito, H. Ohta, H. Terasaki, Y. Katoh, H. Nagashima, K. Jinno, K. Itoh, R. D. Trengove, J. Harrowfield, and S. F. Y. Li, J. High Resolut. Chromatogr., 1996, 19, 475.

14. T. Kimura, H. Ohta, K. Wada, K. Jinno, I. Ueta, and Y. Saito, Chromatographia, 2013, 76, 921.

15. K. Yogo, C. Takemura, Y. Saito, and K. Jinno, Anal. Sci., 2011, 27, 1257.

16. Y. Saito, H. Ohta, and K. Jinno, J. Sep. Sci., 2003, 26, 225.

17. K. Ban, Y. Saito, and K. Jinno, Anal. Sci., 2004, 20, 1403.

18. K. Ban, Y. Saito, and K. Jinno, Anal. Sci., 2005, 21, 397.

19. N. S. Quiming, N. L. Denola, I. Ueta, Y. Saito, S. Tatematsu, and K. Jinno, Anal. Chim. Acta, 2007, 598, 41.

20. K. Yogo, N. S. Quiming, Y. Saito, and K. Jinno, Chromatographia, 2009, 70, 677.

21. M. Harper, J. Chromatogr., A, 2000, 885, 129.

22. R. Barro, J. Regueiro, M. Llompart, and C. Garcia-Jares, $J$. Chromatogr., A, 2009, 1216, 540.

23. M. R. Ras, F. Borrull, and R. M. Marcé, TrAC, Trends Anal. Chem., 2009, 28, 347. 
24. J. Pawliszyn, "Solid-Phase Microextraction: Theory and Practice", 1997, Wiley-VCH, New York.

25. H. Kataoka, Anal. Sci., 2011, 27, 893.

26. H. L. Lord, W. Zhan, and J. Pawliszyn, Anal. Chim. Acta, 2010, 677, 3.

27. Y. Saito, I. Ueta, M. Ogawa, A. Abe, K. Yogo, S. Shirai, and K. Jinno, Anal. Bioanal. Chem., 2009, 393, 861.

28. I. Ueta and Y. Saito, Bunseki Kagaku, 2011, 60, 833.

29. Y. Saito, I. Ueta, M. Ogawa, and K. Jinno, "Needle-Trap Devices for Environmental Sample Preparation", in "Comprehensive Sampling and Sample Preparation", ed. J. Pawliszyn, 2012, Chap. 3.45, Elsevier Science B.V., Amsterdam.

30. P. Trefz, S. Kischkel, D. Hein, E. S. James, J. K. Schubert, and W. Miekisch, J. Chromatogr., A, 2012, 1219, 29.

31. Y. Saito, I. Ueta, K. Kotera, M. Ogawa, H. Wada, and K. Jinno, J. Chromatogr., A, 2006, 1106, 190.

32. M. Alonso, A. Godayol, E. Antico, and J. M. Sanchez, J. Sep. Sci., 2011, 34, 2705.

33. A. Wang, F. Fang, and J. Pawliszyn, J. Chromatogr., A, 2005, 1072, 127.

34. M. Mieth, S. Kischkel, J. K. Schubert, D. Hein, and W. Miekisch, Anal. Chem., 2009, 81, 5851.

35. M. Mieth, J. K. Schubert, T. Gröger, B. Sabel, S. Kischkel, P. Fuchs, D. Hein, R. Zimmermann, and W. Miekisch, Anal. Chem., 2010, 82, 2541.

36. J. D. Crom, S. Claeys, A. Godayol, M. Alonso, E. Anticó, and J. M. Sanchez, J. Sep. Sci., 2010, 33, 2833.

37. M. Alonso, L. Cerdan, A. Godayol, E. Anticó, and J. M. Sanchez, J. Chromatogr., A, 2011, 1218, 8131.

38. D. W. Lou, X. Lee, and J. Pawliszyn, J. Chromatogr., A, 2008, 1201, 228.

39. Y. Saito, I. Ueta, M. Ogawa, M. Hayashida, and K. Jinno, J. Pharm. Biomed. Anal., 2007, 44, 1.

40. I. Ueta, Y. Saito, K. Teraoka, T. Miura, and K. Jinno, Anal. Sci., 2010, 26, 569.

41. I. Ueta, Y. Saito, K. Teraoka, H. Matsuura, K. Fujimura, and K. Jinno, Anal. Sci., 2010, 26, 1127.

42. I. Ueta, A. Mizuguchi, K. Fujimura, S. Kawakubo, and Y. Saito, Anal. Chim. Acta, 2012, 746, 77.

43. M. Inoue, A. Mizuguchi, I. Ueta, K. Takahashi, and Y. Saito, Anal. Sci., 2013, 29, 519.

44. I. Ueta, Y. Saito, M. Hosoe, M. Okamoto, H. Ohkita, S. Shirai, H. Tamura, and K. Jinno, J. Chromatogr., B, 2009, 877, 2551.

45. R. Kubinec, V. G. Berezkin, R. Górová, G. Addová, H. Mračnová, and L. Soják, J. Chromatogr., B, 2004, 800, 295.

46. H. Jurdáková, R. Kubinec, M. Jurčišinová, Z. Krkošová, J. Blaško, I. Ostrovský, L. Soják, and V. G. Berezkin, J. Chromatogr., A, 2008, 1194, 161.

47. I. Ueta, N. A. Razak, A. Mizuguchi, S. Kawakubo, Y. Saito, and K. Jinno, J. Chromatogr., A, 2013, 1317, 211.

48. V. G. Berezkin, E. D. Makarov, and B. V. Stolyarov, J. Chromatogr., A, 2003, 985, 63.

49. P. Trefz, L. Rösner, D. Hein, J. K. Schubert, and W. Miekisch, Anal. Bioanal. Chem., 2013, 405, 3105.

50. Y. Saito, A. Tahara, M. Imaizumi, T. Takeichi, H. Wada, and K. Jinno, Anal. Chem., 2003, 75, 5525.

51. Y. Saito, M. Ogawa, M. Imaizumi, K. Ban, A. Abe, T. Takeichi, H. Wada, and K. Jinno, Anal. Bioanal. Chem., $\mathbf{2 0 0 5}, 382,825$.

52. A. Abe, Y. Saito, I. Ueta, K. Nakane, T. Takeichi, and K. Jinno, J. Chromatogr., A, 2009, 1216, 7456.

53. K. Nakane, S. Shirai, Y. Saito, Y. Moriwake, I. Ueta, M. Inoue, and K. Jinno, Anal. Sci., 2011, 27, 811.

54. Y. Saito, A. Tahara, M. Ogawa, M. Imaizumi, K. Ban, H. Wada, and K. Jinno, Anal. Sci., 2004, 20, 335.

55. A. Abe, Y. Saito, M. Imaizumi, M. Ogawa, T. Takeichi, and K. Jinno, J. Sep. Sci., 2005, 28, 2413.

56. S. Shirai, Y. Saito, Y. Sakurai, I. Ueta, and K. Jinno, Anal. Sci., 2010, 26, 1011.

57. Y. Saito, I. Ueta, M. Ogawa, and K. Jinno, Anal. Bioanal. Chem., 2006, 386, 725.

58. I. Ueta, Y. Saito, N. B. A. Ghani, M. Ogawa, K. Yogo, A. Abe, S. Shirai, and K. Jinno, J. Chromatogr., A, 2009, 1216, 2848.

59. I. Y. Eom, V. H. Niri, and J. Pawliszyn, J. Chromatogr., A, 2008, 1196 - 1197, 10.

60. M. Ogawa, Y. Saito, I. Ueta, and K. Jinno, Anal. Bioanal. Chem., 2007, 388, 619.

61. M. Ogawa, Y. Saito, S. Shirai, Y. Kiso, and K. Jinno, Chromatographia, 2009, 69, 685.

62. K. Jinno, M. Ogawa, I. Ueta, and Y. Saito, TrAC, Trends Anal. Chem., 2007, 26, 27.

63. Y. Saito, M. Kawazoe, M. Imaizumi, Y. Morishima, Y. Nakao, K. Hatano, M. Hayashida, and K. Jinno, Anal. Sci., 2002, $18,7$.

64. M. Inoue, Y. Saito, I. Ueta, T. Miura, H. Ohkita, K. Fujimura, and K. Jinno, Anal. Sci., 2010, 26, 687.

65. I. Ueta, K. Takahashi, and Y. Saito, Anal. Sci., 2012, 28, 953.

66. I. Ueta, A. Mizuguchi, K. Tani, S. Kawakubo, and Y. Saito, Anal. Sci., 2013, 29, 673.

67. K. Jinno, Y. Saito, and M. Imaizumi, Bunseki Kagaku, 2001, 50, 775.

68. Y. Saito, K. Jinno, and T. Greibrokk, J. Sep. Sci., 2004, 27, 1379.

69. Y. Saito, M. Ogawa, M. Imaizumi, K. Ban, A. Abe, T. Takeichi, H. Wada, and K. Jinno, J. Chromatogr. Sci., 2005, 43, 536.

70. M. Ogawa, Y. Saito, M. Imaizumi, H. Wada, and K. Jinno, Chromatographia, 2006, 63, 459. 